\title{
Generation of synthetic datasets for transformer's dissolved gas analysis using Monte-Carlo Simulation
}

\author{
Eaby Kollonoor Babu \\ Dept. of Computing and Information \\ Sciences \\ Northumbria University \\ Newcastle, UK \\ eaby.k.babu@northumbria.ac.uk
}

\author{
Imran Bashir \\ School of Computing, Engineering and \\ Information Sciences \\ Teesside University \\ Middlesbrough, UK \\ I.Bashir@tees.ac.uk
}

\author{
Gobind Pillai \\ School of Computing, Engineering and \\ Information Sciences \\ Teesside University \\ Middlesbrough, UK \\ g.g.pillai@tees.ac.uk
}

\author{
Kiran Chandrakumar Jyothi \\ Kent Business School \\ University of Kent \\ Gillingham, UK \\ kc603@kent.ac.uk
}

\begin{abstract}
The fault diagnosis in power transformers is carried out using Dissolved Gas Analysis (DGA). Although DGA does provide key information for fault detection, the method is inherently complex. Several methods have been developed for DGA, but still possess challenges in accurately detecting the fault. A method has been developed to generate synthetic data using Monte-Carlo simulation. The generated synthetic data is feed into DGA excel tool to investigate the accuracy of fault detection. The synthetic data can be used to further enhance the DGA tool, improve its accuracy and investigate the inclusive faults. A model has been proposed for the integration of synthetic data generator with DGA tool for machine learning and to obtain an automated and improved DGA tool for fault diagnoses in power transformers.
\end{abstract}

Keywords-Power transformers, fault diagnosis, dissolved gas analysis, Monte-Carlo simulation

\section{INTRODUCTION}

Power transformers are an integral part of any electrical power network and it's essential to have the means for early fault detection and preventive maintenance in them. Dissolved Gas Analysis (DGA) is a common method that is in use currently for incipient fault diagnosis. Certain gases are generated because of the thermal and electrical stress produced in the transformer's insulating oil (mineral oil) during its prolonged operation. Faults in the transformer can drastically increase the generation rates of these gases in certain characteristic ways. The relevant gases generated are hydrogen $(\mathrm{H} 2)$, methane $(\mathrm{CH} 4)$, acetylene $(\mathrm{C} 2 \mathrm{H} 2)$, ethylene (C2H4), and ethane (C2H6). The decomposition of the insulating paper results in the occurrence of carbon monoxide (CO) and carbon dioxide $(\mathrm{CO} 2)$ in addition $[1,2]$. DGA involves analyzing the concentration of these gases in the transformer oil and comparing it with historical data to find the gas generation rates. Certain ratios of the gas concentrations are calculated which can help us with identifying the fault type. The many approaches developed for analyzing these gases and interpreting their significance include Key Gas, Dornenburg Ratio, Rogers Ratio, Nomograph, IEC Ratio, Duval Triangle, and CIGRE [3].

DGA has traditionally been done manually but many ways to automate the process can be found in the literature. A diagnostic tool based on MS Excel spreadsheet has been produced in [3]. An expert model based on the fuzzy logic approach to finding the transformer incipient fault in [5]. A
Support Vector Machine (SVM) with six classifiers and minor training data has been used to do four DGA methods and provide the appropriate result in [6]. A similar method in which the wavelet technique is combined with least-squares SVM has been studied in [7]. [8] proposes the use of a DGA diagnostic tool based on Artificial Neural networks (ANN) for more accurate fault detection in transformers with multiple faults. A Neural Pattern Recognition (NPR) has been suggested in [9] which uses different data transformation techniques to improve train the Artificial Intelligence (AI) the diagnostic accuracy of the model. The main drawbacks with these AI-based methods are the huge costs involved and the high amounts of time and computing power required to train the model to start producing accurate results. The results produced in these works are from limited DGA data sets that are available in the public domain, which is not enough to sufficiently train the models. Hence, it is proposed in this paper the use of synthetic data sets to train the proposed DGA tool, which can be created using synthetic data generation techniques.

Synthetic data generation has been used before in recent literature to create datasets for model training. A machinelearning-based synthetic data generation method has been used in [10] for healthcare applications, which generates synthetic time-series data that composed of nested sequences using hidden Markov models and regression models. A complex nested model has been utilized to create terabytes of structurally similar data for Internet of Things (IoT) research by the authors in [11]. The application of synthetic data generation to create training datasets can be observed in many other fields such as in plasma current quench studies [12], to analyze and predict seismic activities [13], to correctly identify and detect people using omnidirectional cameras [14], wastewater treatment modeling studies [15], and meteorological studies $[16,17]$. The synthetic data has been used to test the DGA toolbox. The accuracy of DGA toolbox has been investigated using synthetic data along with its applications for machine learning.

DGA is important for the security, reliability, maintenance, and economic operation of power system infrastructure. It is evident from the literature review that there is a knowledge gap when it comes to online / machine learning-based DGA methods and one of the obstacles in the way of addressing this is the lack of synthetic data sets. Therefore, the objective of this paper is to address the gap by 
developing a method for generating synthetic dissolved gas data for DGA and validating it.

The rest of the paper is organized as follows: Section II explains the methodology used for synthetic data generation and its segregation, Section III discusses the use of machine learning model implementation, Section IV is the discussion of the results and Section V is the Conclusion.

\section{Methodology}

For DGA, it is important to identify and interpret the fault correctly. Multiple DGA methods have been developed, but still discrepancies exist in detecting the fault. Duval triangle and Pentagon methods were developed to reach a conclusive decision. Nevertheless, there still exists some unidentified fault. Therefore, the developed DGA toolbox is being trained using synthetic data. The synthetic data is generated to identify all possible faults with a focus on fault regimes, where a conclusive decision cannot be taken.

The procedure for data synthesis mainly consists of 2 stages. In stage one we find the data pattern from a preexisting dataset of DGA's. This data is passed to a data segregator block which understands the data structure and finds the number of classes, upper limit, lower limit, mean, median, variant, mode, standard deviation, degree of freedom, and range from any given numerical dataset. These values are stored in a data collector block which acts as an input to the Monte-Carlo data generator in stage 2 of the process. The block diagram of the proposed process is shown below.

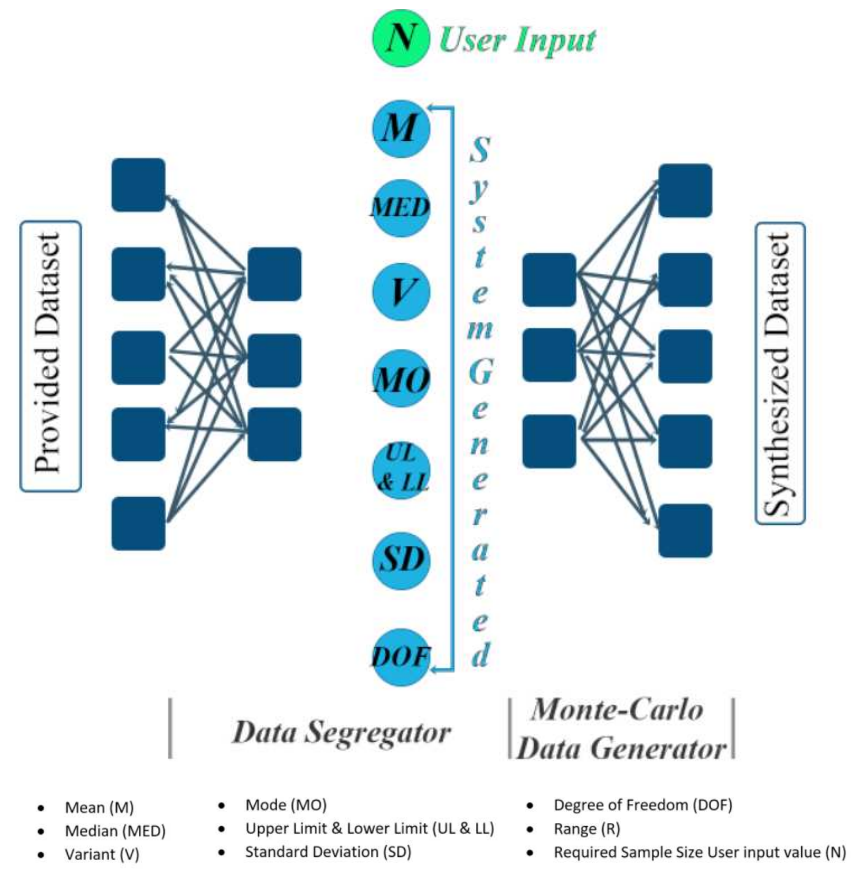

Fig. 1. Block diagram for data synthesis

\section{A. Data Segregator}

This functional block takes any numerical dataset as input and understands the structure of the dataset provided. This is carried out with 3 functions stated below.

Class Finder: From the provided dataset this function finds all the column header and checks for numerical value content in each column, if multiple numerical values are found it makes a class with the column header name. This process is carried out till all columns are scanned. Once this scanning is completed the Class finder passes the class names to the data collector class.

Limit Finder: For each of the found classes this function finds the upper limit and lower limit after analyzing each of the class data. The formula used in the limit finder is:

$$
\begin{gathered}
f_{\text {Max }}=\operatorname{MAX}\left(A 2: A_{n}\right) \\
f_{\text {min }}=\operatorname{MIN}\left(A 2: A_{n}\right)
\end{gathered}
$$

Stat Generator: Once all the classes are passed to the Data Collector, we then use Stat Generator to get mean, median, variant, mode, range, standard deviation, degree of freedom, and number repeatability.

For finding the median of the series and Degree of Freedom, we use a different approach which is as below;

$$
f_{M E D}= \begin{cases}A\left[\frac{n}{2}\right] & \text { if } n \text { is even } \\ \frac{A\left[\frac{n-1}{2}\right]+A\left[\frac{n+1}{2}\right]}{2} & \text { if } n \text { is odd }\end{cases}
$$

And for Degree of Freedom the formula is;

$$
D F=n-1 \quad \text { for each of the class series }
$$

Once all the above paraments are calculated the results are passed to the Data Collector. The Data Collector as the input to the Monte-Carlo data generator.

\section{B. Monte-Carlo Data Generator}

The algorithm for the proposed Mote-Carlo generator is given as,

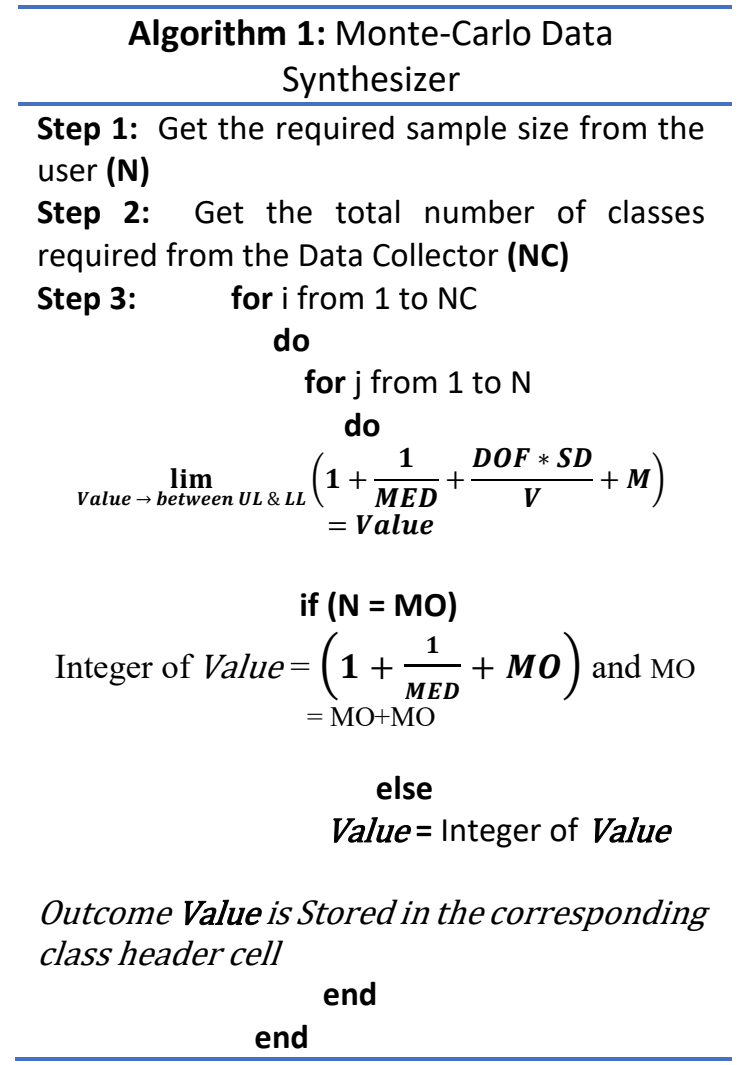


will improve significantly. Moreover, the proposed models in Figure 3 and Figure 4 are a step forward to implement the DGA toolbox in Industry 4.0.

\section{RESULTS AND DISCUSSION}

The main reason for using synthetic data for DGA study is because it's not easy to obtain large sets of real data. Therefore, the expected boundaries of data have been used to generate synthetic data using the Monte-Carlo Data generator. DGA excel tool has been feed-in into synthetic data to study the accuracy and inclusive faults [18].

Synthetic data is used to study the accuracy of DGA faults as given in Table I. Figure 2 plots the accuracy of the predicted faults by DGA tool for synthetic data input as generated by Monte-Carlo simulations. For 70 data points generated for Thermal faults exceeding $700^{\circ} \mathrm{C}$ (T3) by the synthetic data generator, the excel tool predicted 65 T3 faults accurately, with an accuracy of $92.8 \%$. Similarly, the error was calculated for all other faults. It has been calculated that the Discharge of low energy (D1) has the highest accuracy of $93.33 \%$, followed by T1 and T3 at 92\%, then PD at 90\%, T2, $\mathrm{D} 2$, and $\mathrm{S}$ stands at $86 \%$. The synthetic data generator was able to generate a dataset for all the desired faults as the user's requirement with an overall accuracy of above $90 \%$.

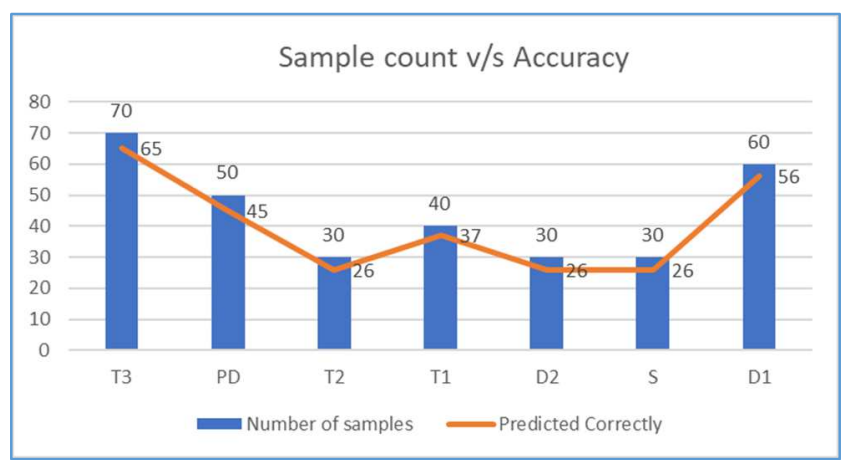

Fig. 4. Accuracy of the predicted faults by DGA tool for synthetic data input as generated by Monte-Carlo simulations.

Several simulations have been carried out to test the accuracy of DGA excel tool for different sets of synthetic data generation. The accuracy of the fault diagnosis remained above $86 \%$. It has been concluded that the DGA tool and synthetic data generator is shown the results as expected. The DGA excel tool be integrated with a synthetic data generator for machine learning and artificial intelligence applications.

\section{CONCLUSIONS}

DGA is important for the security, reliability, maintenance, and economic operation of power system infrastructure. In this paper, we developed a method for generating synthetic dissolved gas data for DGA using MonteCarlo Simulation. In the previous work, an excel tool was developed for DGA which was used in this work to validate the synthetic data. The synthetic data generator and DGA tool are integrated and the accuracy of the synthetic data has been investigated. It has been concluded that for all fault detection cases using synthetic data, accuracy of more than $85 \%$ has been achieved. The synthetic data can be used to further enhance the DGA tool, improve its accuracy and investigate the inclusive faults. A model has also been proposed to integrate the synthetic data generator with DGA tool for machine learning applications. This model can help to train machine learning by generating several different synthetic datasets. Further work will be a focus on the implementation of the proposed model for machine learning applications.

\section{REFERENCES}

[1] N.A. Bakar, A. Abu-Siada, and S. Islam, "A review of dissolved gas analysis measurement and interpretation techniques", IEEE Electri. Insul. Mag., pp. 39-49, May 2014.

[2] H. de Faria Jr., J. G. S. Costa and J. L. M. Olivas, "A review of monitoring methods for predictive maintenance of electric power transformers based on dissolved gas analysis", Ren. Sust. Energy Rev., vol. 46, pp.201-209, June 2015.

[3] H. Sun, Y. Huang and C. Huang, "A review of dissolved gas analysis in power transformers", Energy Procedia, vol. 12, pp. 1220-1225, March 2012.

[4] A. Mekkayil, G. Pillai and M. Malcolm, "A spreadsheet based dissolved gas analysis diagnostic tool for oil-filled transformers", 55th Int. Univ. Power Eng. Conf., September 2020.

[5] A. Abu-Siada and S. Hmood, "A new fuzzy logic approach to identify power transformer criticality using dissolved gas-in-oil analysis", Int. J. Elec. Power \& Ener. Sys., vol. 67, pp. 401-408, May 2015.

[6] K. Bacha, S. Souahlia and M. Gossa, "Power transformer fault diagnosis based on dissolved gas analysis by support vector machine", Elec. Power Sys. Res., vol. 83, pp. 73-79, February 2012.

[7] H. Zheng et al., "A novel model based on wavelet LS-SVM integrated improved PSO algorithm for forecasting of dissolved gas contents in power transformers", Elec. Power Sys. Res., vol. 155, pp. 196-205, February 2018.

[8] G. S. Naganathan et al., "Internal fault diagnosis of power transformer using artificial neural network", Materials Today: Proceedings, March 2021.

[9] I. B. M. Taha, S. S. Dessouky and S. S. M. Ghoneim, "Transformer fault types and severity class prediction based on neural patternrecognition techniques", Elec. Power Sys. Res., vol. 191, February 2021.

[10] J. Dahmen and D. Cook, "SynSys: A synthetic data generation system for healthcare applications", Sensors, vol. 19, March 2019.

[11] J. W. Anderson, K. E. Kennedy, L. B. Ngo, A. Luckow and A. W. Apon, "Synthetic data generation for the internet of things", 2014 IEEE Int. Conf. Big Data, pp. 171-176, October 2014.

[12] N. Dalsania, Z. Patel, S. Purohit and B. Choudhary, "An application of machine learning for plasma current quench studies via synthetic data generation”, Fusion Eng. Des., vol. 171, April 2021.

[13] G. Roncoroni, C. Fortini, L.Bortolussi, N.Bienati and M. Pipan, "Synthetic seismic data generation with deep learning", J. App. Geophysics, vol. 190, April 2021.

[14] N. Aranjuelo, S. Garcia, E. Loyo, L. Unzueta and O. Otaegui, "Key strategies for synthetic data generation for training intelligent systems based on people detection from omnidirectional cameras", Comp. Elec. Engg., vol. 192, March 2021.

[15] L.J.P.Snip, X. Flores-Alsina, I. Aymerich, S. Rodríguez-Mozaz, D. Barceló, B. G. Plósz, Ll. Corominas, I. Rodriguez-Roda, U. Jeppsson and K.V. Gernaeya, "Generation of synthetic influent data to perform (micro)pollutant wastewater treatment modelling studies", Sci. Tot. Env., vol. 569-570, pp. 278-290, November 2016.

[16] M. A. Hassan, M. Abubakr and A. Khalil, "A profile-free nonparametric approach towards generation of synthetic hourly global solar irradiation data from daily totals", Renewable Energy, vol. 167, pp. 613-628, April 2021.

[17] M. Bervida, L. Patruno, S. Stanic and S. de Miranda, "Synthetic generation of the atmospheric boundary layer for wind loading assessment using spectral methods", J. Wind Engg. Ind. Aero., vol. 196, January 2020.

[18] A. M. Berg, S. T. Mol, G. Kismihok, and N. Sclater, "The Role of a Reference Synthetic Data Generator within the Field of Learning Analytics," J. Learn. Anal., vol. 3, no. 1, pp. 107-128, 2016. 